\title{
Application of self-organizing map to failure modes and effects analysis methodology
}

\author{
Wui Lee Chang, Lie Meng Pang, Kai Meng Tay*
}

Faculty of Engineering, Universiti Malaysia Sarawak, Sarawak, Malaysia

\section{A R T I C L E I N F O}

\section{Article history:}

Received 23 September 2015

Revised 3 December 2015

Accepted 10 April 2016

Available online $\mathrm{xxx}$

\section{Keywords:}

Failure modes and effects analysis

Clustering

Visualization

Self-organizing map

Risk Priority Number Interval

\begin{abstract}
A B S T R A C T
In this paper, a self-organizing map (SOM) neural network is used to visualize corrective actions of failure modes and effects analysis (FMEA). SOM is a popular unsupervised neural network model that aims to produce a low-dimensional map (typically a two-dimensional map) for visualizing high-dimensional data. With regards to FMEA, it is a popular methodology to identify potential failure modes for a product or a process, to assess the risk associated with those failure modes, also, to identify and carry out corrective actions to address the most serious concerns. Despite the popularity of FMEA in a wide range of industries, two well-known shortcomings are the complexity of the FMEA worksheet and its intricacy of use. To the best of our knowledge, the use of computation techniques for solving the aforementioned shortcomings is limited. The use of SOM in FMEA is new. In this paper, corrective actions in FMEA are described in their severity, occurrence and detect scores. SOM is then used as a visualization aid for FMEA users to see the relationship among corrective actions via a map. Color information from the SOM map is then included to the FMEA worksheet for better visualization. In addition, a Risk Priority Number Interval is used to allow corrective actions to be evaluated and ordered in groups. Such approach provides a quick and easily understandable framework to elucidate important information from a complex FMEA worksheet; therefore facilitating the decision-making tasks by FMEA users. The significance of this study is two-fold, viz., the use of SOM as an effective neural network learning paradigm to facilitate FMEA implementations, and the use of a computational visualization approach to tackle the two well-known shortcomings of FMEA.
\end{abstract}

(c) 2017 Elsevier B.V. All rights reserved.

\section{Introduction}

Failure modes and effects analysis (FMEA) is an effective problem prevention and risk analysis methodology for defining, identifying, and eliminating failures of a system, design, process, or service [1]. A search in the literature reveals that FMEA was extensively used in a wide range of application domains, e.g., aerospace [2], automotive [1], nuclear [3], electronic [4], manufacturing [5,6], chemical [7], mechanical [8], healthcare and hospital [9], and agriculture [10]. FMEA usually starts with identifying the failure modes of a system or process, understanding the causes and effects of each failure mode, and determining suitable corrective actions to eliminate or reduce the risk of the respective failure modes [1]. Traditionally, the risk of a failure mode is determined by a Risk Priority Number (RPN) model [1]. The RPN model considers three risk factors as its inputs, i.e. severity (S), occurrence (O), and detection (D), and produces an RPN score (i.e. multiplication of $\mathrm{S}$, $\mathrm{O}$, and $\mathrm{D}$ ) as the output [1]. $\mathrm{S}$ and $\mathrm{O}$ are seriousness and frequency

\footnotetext{
* Corresponding author.

E-mail addresses: kmtay@unimas.my, tkaimeng@yahoo.com (K.M. Tay).
}

of a failure mode and its root cause(s), respectively, while D is the effectiveness of the existing measures in detecting a failure mode before the effect of the failure mode reaches the customer(s) [1].

While the effectiveness of FMEA has been demonstrated, three shortcomings pertaining to practical implementation of FMEA are as follows. (1) its risk evaluation and prioritization issues [2,5,11,12]; (2) the complexity of the FMEA worksheet [13]; and (3) its intricacy of use $[13,14]$. The first shortcoming is well known and much research works have been conducted $[2,11]$. The first shortcoming suggests that the traditional RPN model is susceptible to a number of limitations, among the popular are, (1) relative importance among $\mathrm{S}, \mathrm{O}$ and $\mathrm{D}$ is not taken into consideration [2], (2) different combinations of S, O and D may produce exactly the same value of RPN, but their hidden risk implications may be totally different [5], (3) the three risk factors are difficult to be precisely evaluated [11], (4) the mathematical formula for calculating RPN is questionable [11] and etc. Besides, according to a review from [11], the existing risk evaluation methods can be grouped into five categories, i.e., multi-criteria decision making methods, mathematical programming methods, artificial intelligence methods, integrated methods, and other methods. 